\title{
Supersymmetry in string bit models with internal degrees of freedom
}

\author{
Charles B. Thorn ${ }^{*}$ \\ Institute for Fundamental Theory, Department of Physics, University of Florida, \\ Gainesville, Florida 32611, USA
}

(Received 19 December 2019; accepted 27 January 2020; published 14 February 2020)

\begin{abstract}
We study in s general way the construction of string bit Hamiltonians which are supersymmetric, We construct several quadratic and quartic polynomials in string bit creation and annihilation operators $\bar{\phi}_{a_{1} \cdots a_{n}}^{A}, \phi_{a_{1} \cdots a_{n}}^{A}$, which commute with the supersymmetry generators $Q^{a}$. Among these operators are ones with the spinor tensor structure required to provide the lightcone worldsheet vertex insertion factors needed to give the correct interactions for the IIB superstring, whenever a closed string separates into two closed strings or two closed strings join into one.
\end{abstract}

DOI: 10.1103/PhysRevD.101.046017

\section{INTRODUCTION}

The aim of string bit models [1] is to provide a bona fide quantum mechanical system whose dynamics leads, in a certain limit, to string theory. Since most consistent versions of string theory in a Poincare invariant background contain a massless spin 2 particle, a successful string bit model could also provide a bona fide quantum theory of gravity.

The central premise underlying string bit models is that spacetime is, at least in part, an emergent concept. This idea has close parallels to 't Hooft's proposal that the world is a hologram [2]. String bit models developed from an early proposal to define the worldsheet system of string theory on a lightcone lattice: Choose lightcone parametrization $[3,4]$, $\tau=x^{+}, \mathcal{P}^{+}=1$, where for a Lorentz vector $V^{\mu}, V^{ \pm}=$ $\left(V^{0} \pm V^{1}\right) / \sqrt{2}$ and $\mathcal{P}^{\mu} d \sigma$ is the Lorentz momentum contained in the element $d \sigma$ of string, and put the resultant system on a rectangular $\sigma, \tau$ lattice [5]. Because of the way $\sigma$ has been fixed, each lattice site is assigned exactly one unit $m$ of $P^{+}$. Then a little reflection shows that the lattice system, after taking the continuum limit of the time parameter, defines a quantum system of $M=P^{+} / m$ particles (string bits), ordered on a line, moving in the transverse space only. Their dynamics is Galilei invariant with $m$ playing the role of Newtonian mass. These simple observations already show that $P^{+}$is emergent: it is simply the total Newtonian mass of a chain of a very large number

\footnotetext{
*thorn@phys.ufl.edu
}

Published by the American Physical Society under the terms of the Creative Commons Attribution 4.0 International license. Further distribution of this work must maintain attribution to the author(s) and the published article's title, journal citation, and DOI. Funded by SCOAP. of string bits. Its conjugate $x^{-}$"emerges" from the string bit dynamics.

However, there is an aspect of the story just told that is artificial. The ordering of the string bits on a chain is by fiat. If the string bits are indeed independent fundamental entities, there should be interactions between all pairs of bits, not just the nearest neighbors of bits ordered on a chain. In other words, we should be able to describe the presence of a string bit in the language of second quantization by the action of a creation operator $\phi^{\dagger}$ on the empty state $|0\rangle$, with the dynamics given by the Hamiltonian as a function of creation and annihilation operators. 't Hooft has identified a way to enhance chain ordering by taking a large $N$ limit [6]. Promote the bit annihilation operator to an $N \times N$ matrix operator $\phi_{\alpha}^{\beta}$. Then a Hamiltonian with the structure $N^{-1} \operatorname{Tr} \phi^{\dagger} \phi^{\dagger} \phi \phi$ describes chains of bits with nearest neighbor interactions in the limit $N \rightarrow \infty$ [7]. Taking $N$ large rigs the dynamics in favor of chain formation. But if the nearest neighbor interactions are not sufficient to make a long chain of bits stable against decay into smaller chains, continuous strings would not form. Indeed, the tachyonic instability of the bosonic string can be traced to such a failure.

As shown by the stability of superstring [8-10], one cure for such an instability is to include a mix of bosonic and fermionic string bits [11]. In recent work on these models [12-17] and in this article, we adorn the string bit annihilation operator with three types of indices

$$
\left(\phi_{\left[a_{1} \cdots a_{n}\right]}^{A}\right)_{\alpha}^{\beta} .
$$

The Greek letters $\alpha, \beta=1, \ldots, N$ are color indices labeling the matrix elements of the matrix operators, whose function is to provide a way to favor nearest neighbor chain interaction. The Hamiltonian is assumed to be invariant 
under color $S U(N)$. Since the adjoint of $\left(\phi^{A}\right)_{\beta}^{\alpha}$ has the color transformation properties of $\left(\phi^{A}\right)_{\alpha}^{\beta}$ it is convenient to define the creation operator $\left(\bar{\phi}^{A}\right)_{\alpha}^{\beta}=\left(\left(\phi^{A}\right)_{\beta}^{\alpha}\right)^{\dagger}$.

Lower case Latin letters $a_{k}=1, \ldots, s$. are spin indices, with the number of spin indices $n=0,1, \ldots, s$. Each operator is completely antisymmetric under permutations of the $a$ 's. There are $2^{s}$ distinct sets of these indices. The Hamiltonian is assumed to be invariant under $O(s)$ rotations of these indices. For generic $s$ these are vector indices, but for some special values of $s$, they may alternatively be taken as spinor indices. Whether or not the string bit system leads to a Poincaré invariant string theory we specify that $\phi$ satisfies commutation relations when $n$ is even and anticommutation relations when $n$ is odd. For $s>0$ there are an equal number of bosonic and fermionic annihilation operators. With this assignment of statistics, the spin statistics theorem implies that only values of $s$ that admit spinor representations can lead to the emergence of Poincare invariance. In the case of principal interest for string theory, $s=8$ admits the $\mathrm{SO}(8)$ spinors comprising the physical Majorana-Weyl degrees of freedom (d.o.f.) required of the $D=10$ superstring. In the case of the protostring $s=24$ can be taken as $3 S O(8)$ Majorana spinors.

Finally, the capital Latin superscript $A=1, \ldots, N_{f}$ is a label for internal (nonspin or "flavor") d.o.f. For the purposes of this article we make no assumptions about their structure. In [13] it was shown how to choose their dynamics so as to generate a Heisenberg spin system on a chain of bits, which simulates noncompact transverse coordinates. Such a choice would presumably be important for precisely inducing the superstring, but in this article we leave their structure arbitrary. We have borrowed the terms color and flavor from the standard model, because their roles in their respective models are similar: String bits will be permanently confined in color singlet string as quarks and gluons are in color singlet hadrons. And flavor distinguishes otherwise identical string bits as flavor distinguishes otherwise identical quarks and leptons.

In string bit models, supersymmetry plays a fundamental role in the dynamics of string formation. Consider a closed chain of $M$ bits in the limit of very large $M$. Stringy behavior can ensue if there are excitation energies above the ground state of order $O(1 / M)$. This occurs for example if there are vibrational modes and also for ordinary spin waves arising from fluctuations between different spin states of each bit with the same statistics. In both cases the large $M$ behavior of the ground energy has the behavior $E(M) \sim \alpha M+\gamma / M$ with $\gamma<0$. In our string bit models, at order $1 / N$, a closed chain can split into two similar but smaller closed chains such that $M=M_{1}+M_{2}$. Then $\gamma<0$ would mean that the longer chain is unstable to decay into smaller chains (as long as the daughter chains are long enough). This is ultimately the reason the bosonic closed string as well as the Neveu-Schwarz (NS,NS) closed string have tachyonic ground states.

If, however, there are waves arising from fluctuations between bosonic and fermionic states of each string bit, the sign of $\gamma$ reverses and the single string is stable: it requires added energy to transform into two smaller chains. This is what happens in the Green-Schwarz lightcone superstring in which the fermionic worldsheet field $S$ is a rotational spinor in target space i.e., it creates a target space fermionic state from a bosonic one and vice versa. But for such $1 / M$ excitations to be possible each bit must possess a fermionic and a bosonic state of the same energy: there must be at least one Grassmann odd operator $Q$ which commutes with the Hamiltonian. This is all that is meant by supersymmetry in the following. It is a lot less supersymmetry than the full supersymmetry of an emergent superstring, which among other things would require another Grassmann odd operator $R$ such that $H=R^{2}$. This larger supersymmetry is not necessary for stable string formation, but rather is crucial for the Poincare invariance of any emergent string theory. ${ }^{1}$

In this article we explore ways to construct supersymmetric string bit Hamiltonians in the presence of internal nonspin d.o.f. represented by the capital superscript in (1). In Sec. II we recall some essential features of the string bit formalism that we need for this work. The construction of supersymmetric polynomials quadratic and quartic in bit creation and annihilation operators is developed in Sec. III. Finally, in Sec. IV, we conclude by applying the tools developed in Sec. III to build quartic terms, which modify the Hamiltonian of [13] to supply the necessary prefactors to produce superstring scattering amplitudes.

\section{REVIEW OF THE STRING BIT FORMALISM}

The string bit creation and annihilation operators described in the Introduction satisfy the (anti)commutation relations

$$
\begin{aligned}
& {\left[\left(\phi_{a_{1} \cdots a_{m}}^{A}\right)_{\alpha}^{\beta},\left(\bar{\phi}_{b_{1} \cdots b_{n}}^{B}\right)_{\gamma}^{\delta}\right]_{ \pm}} \\
& \quad \equiv\left(\phi_{a_{1} \cdots a_{m}}^{A}\right)_{\alpha}^{\beta}\left(\bar{\phi}_{b_{1} \cdots b_{n}}^{B}\right)_{\gamma}^{\delta}-(-)^{m n}\left(\bar{\phi}_{b_{1} \cdots b_{n}}^{B}\right)_{\gamma}^{\delta}\left(\phi_{a_{1} \cdots a_{m}}^{A}\right)_{\alpha}^{\beta} \\
& \quad=\delta_{A B} \delta_{m n} \delta_{\alpha}^{\delta} \delta_{\gamma}^{\beta} \sum_{P}(-)^{P} \delta_{a_{1} b_{P_{1}}} \cdots \delta_{a_{n} b_{P_{n}}}
\end{aligned}
$$

which incorporate the fact that $\bar{\phi}$ creates a boson if $n$ is even and a fermion if $n$ is odd. The sum over $P$ is over all permutations of $1,2, \ldots, n$.

In [13] we studied a Hamiltonian with a very specific implementation of the dynamics of these internal "flavor" d.o.f. We wrote $H=H_{F}+H_{S}$ with $H_{F}$ diagonal in spin, but nontrivial in flavor, and vice versa for $H_{S}$

\footnotetext{
${ }^{1}$ It is not obvious how much of the symmetry one finds in the emergent string theory should or even can be present in the string bit Hamiltonian. After all the latter makes no reference to space at all, to say nothing of super-Poincaré invariance.
} 
$H_{F}=\frac{2}{N} \sum_{n=0}^{s} \sum_{k=0}^{s} \frac{1}{n ! k !} \operatorname{Tr} \bar{\phi}_{a_{1} \cdots a_{n}}^{A} \bar{\phi}_{b_{1} \cdots b_{k}}^{B} \phi_{b_{1} \cdots b_{k}}^{C} \phi_{a_{1} \cdots a_{n}}^{D} T_{A B C D}$

and for $H_{S}$

$$
H_{S}=H_{1}+H_{2}+H_{3}+H_{4}+H_{5},
$$

where the $H_{i}$ are

$$
\begin{aligned}
& H_{1}=\frac{2}{N} \sum_{n=0}^{s} \sum_{k=0}^{s} \frac{s-2 n}{n ! k !} \operatorname{Tr} \bar{\phi}_{a_{1} \cdots a_{n}}^{A} \bar{\phi}_{b_{1} \cdots b_{k}}^{B} \phi_{b_{1} \cdots b_{k}}^{B} \phi_{a_{1} \cdots a_{n}}^{A}, \\
& H_{2}=\frac{2}{N} \sum_{n=0}^{s-1} \sum_{k=0}^{s-1} \frac{(-)^{k}}{n ! k !} \operatorname{Tr} \bar{\phi}_{a_{1} \cdots a_{n}}^{A} \bar{\phi}_{b b_{1} \cdots b_{k}}^{B} \phi_{b_{1} \cdots b_{k}}^{B} \phi_{b a_{1} \cdots a_{n}}^{A}, \\
& H_{3}=\frac{2}{N} \sum_{n=0}^{s-1} \sum_{k=0}^{s-1} \frac{(-)^{k}}{n ! k !} \operatorname{Tr} \bar{\phi}_{b a_{1} \cdots a_{n}}^{A} \bar{\phi}_{b_{1} \cdots b_{k}}^{B} \phi_{b b_{1} \cdots b_{k}}^{B} \phi_{a_{1} \cdots a_{n}}^{A}, \\
& H_{4}=\frac{2 i}{N} \sum_{n=0}^{s-1} \sum_{k=0}^{s-1} \frac{(-)^{k}}{n ! k !} \operatorname{Tr} \bar{\phi}_{a_{1} \cdots a_{n}}^{A} \bar{\phi}_{b_{1} \cdots b_{k}}^{B} \phi_{b b_{1} \cdots b_{k}}^{B} \phi_{b a_{1} \cdots a_{n}}^{A}, \\
& H_{5}=-\frac{2 i}{N} \sum_{n=0}^{s-1} \sum_{k=0}^{s-1} \frac{(-)^{k}}{n ! k !} \operatorname{Tr} \bar{\phi}_{b a_{1} \cdots a_{n}}^{A} \bar{\phi}_{b b_{1} \cdots b_{k}}^{B} \phi_{b_{1} \cdots b_{k}}^{B} \phi_{a_{1} \cdots a_{n}}^{A} .
\end{aligned}
$$

Torres [18] has shown that both $H_{F}$ and $H_{S}$ commute with the supersymmetry operators

$$
\begin{aligned}
Q^{a} & =\sum_{n=0}^{s-1} \frac{(-)^{n}}{n !} \operatorname{Tr}\left[\gamma \bar{\phi}_{a_{1} \cdots a_{n}}^{A} \phi_{a a_{1} \cdots a_{n}}^{A}+\gamma^{*} \bar{\phi}_{a a_{1} \cdots a_{n}}^{A} \phi_{a_{1} \cdots a_{n}}^{A}\right] \\
\gamma & \equiv e^{i \pi / 4}
\end{aligned}
$$

where $M$ is the bit number operator, $N_{F}$ is the number of flavors, and all repeated indices are summed. We have introduced the complex number $\gamma$ to reduce clutter in subsequent equations. The vanishing commutator of $Q^{a}$ with $H$ guarantees equal numbers of bosonic and fermionic eigenstates, at each energy level with bit number greater than 0 . In this article we will extend this conclusion to more general Hamiltonians.

Using the commutation relations (2), it is straightforward to obtain the action of the $H_{i}$ on single trace states. We recall from [11-13] the definition of superfields

$$
\bar{\psi}^{A}(\theta)=\sum_{k=0}^{s} \frac{1}{k !} \bar{\phi}_{a_{1} \cdots a_{k}}^{A} \theta^{a_{1}} \cdots \theta^{a_{k}}
$$

where $\theta^{a}$ are Grassmann variables, and using the notation

$$
T\left(A_{1}, \theta_{1} ; \cdots ; A_{M}, \theta_{M}\right)=\operatorname{Tr} \bar{\psi}^{A_{1}}\left(\theta_{1}\right), \ldots, \bar{\psi}^{A_{M}}\left(\theta_{M}\right),
$$

we find (suppressing flavor indices)

$$
\begin{aligned}
H_{1} T\left(\theta_{1}, \ldots, \theta_{M}\right)|0\rangle=2 & \sum_{k=1}^{M}\left(s-2 \theta_{k}^{a} \frac{d}{d \theta_{k}^{a}}\right) T\left(\theta_{1}, \ldots, \theta_{M}\right)|0\rangle \\
+ & \frac{2}{N} \sum_{k=1}^{M}\left(s-2 \theta_{k}^{a} \frac{d}{d \theta_{k}^{a}}\right) \sum_{l \neq k, k+1} T\left(\theta_{l} \cdots \theta_{k}\right) T\left(\theta_{k+1} \cdots \theta_{l-1}\right)|0\rangle, \\
H_{2} T\left(\theta_{1}, \ldots, \theta_{M}\right)|0\rangle= & 2 \sum_{k=1}^{M} \theta_{k}^{a} \frac{d}{d \theta_{k+1}^{a}} T\left(\theta_{1}, \ldots, \theta_{M}\right)|0\rangle \\
& +\frac{2}{N} \sum_{k=1}^{M} \sum_{l \neq k, k+1} \theta_{k}^{a} \frac{d}{d \theta_{l}^{a}} T\left(\theta_{l} \cdots \theta_{k}\right) T\left(\theta_{k+1} \cdots \theta_{l-1}\right)|0\rangle, \\
H_{3} T\left(\theta_{1}, \ldots, \theta_{M}\right)|0\rangle= & 2 \sum_{k=1}^{M} \theta_{k+1}^{a} \frac{d}{d \theta_{k}^{a}} T\left(\theta_{1}, \ldots, \theta_{M}\right)|0\rangle \\
& +\frac{2}{N} \sum_{k=1}^{M} \sum_{l \neq k, k+1} \theta_{l}^{a} \frac{d}{d \theta_{k}^{a}} T\left(\theta_{l} \cdots \theta_{k}\right) T\left(\theta_{k+1} \cdots \theta_{l-1}\right)|0\rangle,
\end{aligned}
$$




$$
\begin{aligned}
H_{4} T\left(\theta_{1}, \ldots, \theta_{M}\right)|0\rangle= & -2 i \sum_{k=1}^{M} \theta_{k}^{a} \theta_{k+1}^{a} T\left(\theta_{1}, \ldots, \theta_{M}\right)|0\rangle \\
& -\frac{2 i}{N} \sum_{k=1}^{M} \sum_{l \neq k, k+1} \theta_{k}^{a} \theta_{l}^{a} T\left(\theta_{l} \cdots \theta_{k}\right) T\left(\theta_{k+1} \cdots \theta_{l-1}\right)|0\rangle, \\
H_{5} T\left(\theta_{1}, \ldots, \theta_{M}\right)|0\rangle= & -2 i \sum_{k=1}^{M} \frac{d}{d \theta_{k}^{a}} \frac{d}{d \theta_{k+1}^{a}} T\left(\theta_{1}, \ldots, \theta_{M}\right)|0\rangle \\
& -\frac{2 i}{N} \sum_{k=1}^{M} \sum_{l \neq k, k+1} \frac{d}{d \theta_{k}^{a}} \frac{d}{d \theta_{l}^{a}} T\left(\theta_{l} \cdots \theta_{k}\right) T\left(\theta_{k+1} \cdots \theta_{l-1}\right)|0\rangle .
\end{aligned}
$$

We note that the differential operators are applied to nearest neighbors on the same trace when they involve two distinct Grassmann variables.

The action of the $H_{i}$ on multitrace states takes two forms. When both annihilation operators contract on the same trace, the action can be read off from the preceding formulas. When they act on different traces the action is to fuse them into a single trace as follows:

$$
\begin{aligned}
& H_{1} T\left(\theta_{1} \cdots \theta_{K}\right) T\left(\eta_{1} \cdots \eta_{L}\right)|0\rangle_{\text {Fusion }} \\
& =\frac{2}{N} \sum_{k=1}^{K} \sum_{l=1}^{L}\left(s-2 \theta_{k}^{a} \frac{d}{d \theta_{k}^{a}}\right) T\left(\theta_{k+1} \cdots \theta_{k} \eta_{l} \cdots \eta_{l-1}\right)|0\rangle \\
& \quad+\frac{2}{N} \sum_{k=1}^{K} \sum_{l=1}^{L}\left(s-2 \eta_{l}^{a} \frac{d}{d \eta_{l}^{a}}\right) T\left(\theta_{k} \cdots \theta_{k-1} \eta_{l+1} \cdots \eta_{l}\right)|0\rangle,
\end{aligned}
$$

$$
\begin{aligned}
& H_{2} T\left(\theta_{1} \cdots \theta_{K}\right) T\left(\eta_{1} \cdots \eta_{L}\right)|0\rangle_{\text {Fusion }} \\
& =\frac{2}{N} \sum_{k=1}^{K} \sum_{l=1}^{L} \theta_{k}^{a} \frac{d}{d \eta_{l}} T\left(\theta_{k+1} \cdots \theta_{k} \eta_{l} \cdots \eta_{l-1}\right)|0\rangle \\
& \quad+\frac{2}{N} \sum_{k=1}^{K} \sum_{l=1}^{L} \eta_{l}^{a} \frac{d}{d \theta_{k}} T\left(\theta_{k} \cdots \theta_{k-1} \eta_{l+1} \cdots \eta_{l}\right)|0\rangle,
\end{aligned}
$$

with similar transcriptions for the other $H_{i}$. Here the sequence of labels $k \cdots(k-1)$ means $k \cdots K, 1 \cdots(k-1)$ and similarly for $l \cdots(l-1)$. In each case the differential operators have the same structure as the fission terms, but the states on the right are a suitable pair of single trace states. And when there are two distinct Grassmann operators they act on nearest neighbors on the large trace.

\section{SUPERSYMMETRY WITH FLAVOR}

The bit annihilation operator with flavor has, in addition to spinor indices, another "flavor" index not associated with spin, so we write $\phi_{a_{1} \cdots a_{k}}^{A}$ and we take the supercharge to be given by (10). Note that by construction $Q^{a}$ is Hermitian. Our goal is to construct Hamiltonians which commute with $Q^{a}$ and with $M$. To this end we work out the (anti) commutators $\left([A, B]_{ \pm} \equiv A B \pm B A\right.$, with the + sign chosen only when both $A$ and $B$ are Grassmann odd):

$$
\left[Q^{a}, \bar{\phi}_{b_{1} \cdots b_{k}}^{A}\right]_{ \pm}=(-)^{k} \gamma^{*} \bar{\phi}_{a b_{1} \cdots b_{k}}^{A}+\gamma \sum_{l=1}^{k}(-)^{l+k} \delta_{a b_{l}} \bar{\phi}_{b_{1} \cdots \hat{b}_{l} \cdots b_{k}}^{A},
$$

$$
\left[Q^{a}, \phi_{b_{1} \cdots b_{k}}^{A}\right]_{ \pm}=-\gamma \phi_{a b_{1} \cdots b_{k}}^{A}+\gamma^{*} \sum_{l=1}^{k}(-)^{l+1} \delta_{a b_{l}} \phi_{b_{1} \cdots \hat{b}_{l} \cdots b_{k}}^{A},
$$

where a hat over an index means that index is deleted. The second line follows from the Hermitian conjugate of the first line.

\section{A. Supersymmetry transform of some spinor-tensor bilinears}

For two matrix operators $A, B$ introduce the notation $A \otimes B$ to denote the Hilbert space operator products of independent matrix elements of $A$ and $B$. This notation allows us to suppress color indices in the derivations to follow. When explicit indices are needed we will use the following convention:

$$
(A \otimes B)_{\alpha \gamma}^{\beta \delta} \equiv A_{\alpha}^{\beta} B_{\gamma}^{\delta} .
$$

The Hermitian conjugate of these bilinears is given by

$$
\left[(A \otimes B)_{\alpha \gamma}^{\beta \delta}\right]^{\dagger}=\left(B_{\gamma}^{\delta}\right)^{\dagger}\left(A_{\alpha}^{\beta}\right)^{\dagger}=\bar{B}_{\delta}^{\gamma} \bar{A}_{\beta}^{\alpha}=(\bar{B} \otimes \bar{A})_{\delta \beta}^{\gamma \alpha} .
$$

In addition to taking the trace of $A$ or $B$, one can form a matrix operator from $A \otimes B$ in two ways, which we distinguish by a right arrow over the operator if we contract $\beta$ with $\gamma$ and a left arrow if we contract $\alpha$ with $\delta$. Thus if $C=A \otimes B$ we have

$$
\begin{gathered}
\vec{C}_{\alpha}^{\beta} \equiv C_{\alpha \gamma}^{\gamma \beta}=A_{\alpha}^{\gamma} B_{\gamma}^{\beta}=(A B)_{\alpha}^{\beta}, \\
\overleftarrow{C}_{\alpha}^{\beta} \equiv C_{\gamma \alpha}^{\beta \gamma}=A_{\gamma}^{\beta} B_{\alpha}^{\gamma} .
\end{gathered}
$$


We shall usually choose the first way which corresponds to usual matrix multiplication $A B$. The second way would correspond to $B A$ only if the operator matrix elements all commute. Then we can define several bilinear operators with simple supersymmetry transformation rules. Two bosonic bilinears are

$$
\rho_{A B}=\sum_{n=0}^{s} \frac{1}{n !} \bar{\phi}_{b_{1} \cdots b_{n}}^{A} \otimes \phi_{b_{1} \cdots b_{n}}^{B},
$$

$$
\rho_{A B}^{\prime}=\sum_{n=0}^{s} \frac{n}{n !} \bar{\phi}_{b_{1} \cdots b_{n}}^{A} \otimes \phi_{b_{1} \cdots b_{n}}^{B} .
$$

One must keep in mind that these bilinears have four suppressed color indices, a pair for each of the factor operators. Since $Q^{a}$ is a color singlet one body operator, its commutator with any bilinear leaves these color indices and the ordering of operators undisturbed. Then we calculate the commutators

$$
\begin{aligned}
{\left[Q^{a}, \rho_{A B}\right]=} & \sum_{n=0}^{s} \frac{1}{n !}\left(\left[Q^{a}, \bar{\phi}_{b_{1} \cdots b_{n}}^{A}\right]_{ \pm} \otimes \phi_{b_{1} \cdots b_{n}}^{B}+(-)^{n} \bar{\phi}_{b_{1} \cdots b_{n}}^{A} \otimes\left[Q^{a}, \phi_{b_{1} \cdots b_{n}}^{B}\right]_{ \pm}\right) \\
= & \sum_{n=0}^{s} \frac{1}{n !}\left((-)^{n} \gamma^{*} \bar{\phi}_{a b_{1} \cdots b_{n}}^{A}+\gamma \sum_{l=1}^{n}(-)^{l+n} \delta_{a b_{l}} \bar{\phi}_{b_{1} \cdots \hat{b}_{l} \cdots b_{n}}^{A}\right) \otimes \phi_{b_{1} \cdots b_{n}}^{B} \\
& +\sum_{n=0}^{s} \frac{1}{n !}(-)^{n} \bar{\phi}_{b_{1} \cdots b_{n}}^{A} \otimes\left(-\gamma \phi_{a b_{1} \cdots b_{n}}^{B}+\gamma^{*} \sum_{l=1}^{n}(-)^{l+1} \delta_{a b_{l}} \phi_{b_{1} \cdots b_{l} \cdots b_{n}}^{B}\right) \\
= & \sum_{n=0}^{s}\left(\frac{(-)^{n}}{n !} \gamma^{*} \bar{\phi}_{a b_{1} \cdots b_{n}}^{A} \otimes \phi_{b_{1} \cdots b_{n}}^{B}+\frac{(-)^{n-1}}{(n-1) !} \gamma \bar{\phi}_{b_{1} \cdots b_{n-1}}^{A} \otimes \phi_{a b_{1} \cdots b_{n-1}}^{B}\right) \\
& +\sum_{n=0}^{s}\left(-\frac{(-)^{n}}{n !} \gamma \bar{\phi}_{b_{1} \cdots b_{n}}^{A} \otimes \phi_{a b_{1} \cdots b_{n}}^{B}-\frac{(-)^{n-1}}{(n-1) !} \gamma^{*} \bar{\phi}_{a b_{1} \cdots b_{n-1}}^{A} \otimes \phi_{b_{1} \cdots b_{n-1}}^{B}\right) \\
= & 0
\end{aligned}
$$

after shifting the sum index of the second terms in each set of parentheses $n \rightarrow n+1$.

For $\rho^{\prime}$, we have

$$
\begin{aligned}
{\left[Q^{a}, \rho_{A B}^{\prime}\right]=} & \sum_{n=0}^{s}\left(\frac{(-)^{n}}{(n-1) !} \gamma^{*} \bar{\phi}_{a b_{1} \cdots b_{n}}^{A} \otimes \phi_{b_{1} \cdots b_{n}}^{B}+\frac{n(-)^{n-1}}{(n-1) !} \gamma \bar{\phi}_{b_{1} \cdots b_{n-1}}^{A} \otimes \phi_{a b_{1} \cdots b_{n-1}}^{B}\right) \\
& +\sum_{n=0}^{s}\left(-\frac{(-)^{n}}{(n-1) !} \gamma \bar{\phi}_{b_{1} \cdots b_{n}}^{A} \otimes \phi_{a b_{1} \cdots b_{n}}^{B}-\frac{n(-)^{n-1}}{(n-1) !} \gamma^{*} \bar{\phi}_{a b_{1} \cdots b_{n-1}}^{A} \otimes \phi_{b_{1} \cdots b_{n-1}}^{B}\right) \\
= & \sum_{n=0}^{s} \frac{(-)^{n}}{n !}\left(\gamma \bar{\phi}_{b_{1} \cdots b_{n}}^{A} \otimes \phi_{a b_{1} \cdots b_{n}}^{B}-\gamma^{*} \bar{\phi}_{a b_{1} \cdots b_{n}}^{A} \otimes \phi_{b_{1} \cdots b_{n}}^{B}\right) \\
\equiv & \gamma \eta_{A B}^{a}-\gamma^{*} \bar{\eta}_{A B}^{a} .
\end{aligned}
$$

The next to last line is obtained by writing $n=n-1+1$ in the second terms inside parentheses on the previous two lines and then recognizing that the " $n-1$ " parts cancel against the first terms, leaving the next to last line with the $n$ summation index shifted by 1 .

We can similarly consider the supersymmetry properties of the Grassmann odd bilinears $\eta, \bar{\eta}$, defined in the previous equation

$$
\eta_{A B}^{a}=\sum_{n=0}^{s-1} \frac{(-)^{n}}{n !} \bar{\phi}_{a_{1} \cdots a_{n}}^{A} \otimes \phi_{a a_{1} \cdots a_{n}}^{B} .
$$

Forming $\gamma \eta_{A A}^{a}+\gamma^{*} \eta_{A A}^{a \dagger}$ saturating the color indices as the trace of a matrix product, and summing over all flavors $A$ gives the supercharge $Q^{a}$. Then we calculate 


$$
\begin{aligned}
\left\{Q^{a}, \eta_{A B}^{b}\right\}= & \sum_{n=0}^{s-1} \frac{(-)^{n}}{n !}\left((-)^{n} \gamma^{*} \bar{\phi}_{a b_{1} \cdots b_{n}}^{A}+\gamma \sum_{l=1}^{n}(-)^{l+n} \delta_{a b_{l}} \bar{\phi}_{b_{1} \cdots b_{l} \cdots b_{n}}^{A}\right) \otimes \phi_{b b_{1} \cdots b_{n}}^{B} \\
& +\sum_{n=0}^{s} \frac{1}{n !} \bar{\phi}_{b_{1} \cdots b_{n}}^{A} \otimes\left(-\gamma \phi_{a b b_{1} \cdots b_{n}}^{B}+\gamma^{*} \sum_{l=1}^{n}(-)^{l} \delta_{a b_{l}} \phi_{b b_{1} \cdots \hat{b}_{l} \cdots b_{n}}^{B}\right)+\delta_{a b} \gamma^{*} \sum_{n=0}^{s} \frac{1}{n !} \bar{\phi}_{b_{1} \cdots b_{n}}^{A} \otimes \phi_{b_{1} \cdots b_{n}}^{B} \\
= & \sum_{n=0}^{s-1} \frac{1}{n !}\left(\gamma^{*} \bar{\phi}_{a b_{1} \cdots b_{n}}^{A} \otimes \phi_{b b_{1} \cdots b_{n}}^{B}+n \gamma \bar{\phi}_{b_{1} \cdots b_{n-1}}^{A} \otimes \phi_{a b b_{1} \cdots b_{n-1}}^{B}\right) \\
& +\sum_{n=0}^{s} \frac{1}{n !}\left(-\gamma \bar{\phi}_{b_{1} \cdots b_{n}}^{A} \otimes \phi_{a b b_{1} \cdots b_{n}}^{B}-n \gamma^{*} \bar{\phi}_{b_{1} \cdots b_{n-1}}^{A} \otimes \phi_{b b_{1} \cdots b_{n-1}}^{B}\right)+\delta_{a b} \gamma^{*} \sum_{n=0}^{s} \frac{1}{n !} \bar{\phi}_{b_{1} \cdots b_{n}}^{A} \otimes \phi_{b_{1} \cdots b_{n}}^{B} \\
= & \delta_{a b} \gamma^{*} \sum_{n=0}^{s} \frac{1}{n !} \bar{\phi}_{b_{1} \cdots b_{n}}^{A} \otimes \phi_{b_{1} \cdots b_{n} .}^{B}
\end{aligned}
$$

By taking the Hermitian conjugate, we learn that

$$
\left\{Q^{a}, \eta_{B A}^{b \dagger}\right\}=\delta_{a b} \gamma \sum_{n=0}^{s} \frac{1}{n !} \bar{\phi}_{b_{1} \cdots b_{n}}^{A} \otimes \phi_{b_{1} \cdots b_{n}}^{B}
$$

and then it immediately follows that $\left\{Q^{a}, Q^{b}\right\}=2 M N_{f} \delta_{a b}$ where $M=\sum_{n} \frac{1}{n !} \operatorname{Tr} \bar{\phi}_{b_{1} \cdots b_{n}}^{A} \phi_{b_{1} \cdots b_{n}}^{A}$ is the bit number operator. Another immediate corollary is

$$
\left\{Q^{a}, \gamma \eta_{A B}^{b}-\gamma^{*} \eta_{B A}^{b^{\dagger}}\right\}=0 .
$$

This process of building bilinears can be extended to higher rank spinor-tensors. Consider the bilinears

$$
\Omega_{a_{1} \cdots a_{l}}^{A B, p}=\sum_{k} \frac{(-)^{k l}}{k !} \bar{\phi}_{a_{1} \cdots a_{p} b_{1} \cdots b_{k}}^{A} \otimes \phi_{a_{p+1} \cdots a_{l} b_{1} \cdots b_{k}}^{B}
$$

which include as special cases the bilinears $\rho, \eta^{a}$ and $\bar{\eta}^{a}$ discussed above. In the Appendix, we show that the (anti) commutators of $Q^{c}$ with these bilinears are

$$
\begin{aligned}
{\left[Q^{c}, \Omega_{a_{1} \cdots a_{l}}^{A B, p}\right]_{ \pm}=} & \gamma \sum_{m=1}^{p}(-)^{m+p} \delta_{c a_{m}} \Omega_{a_{1} \cdots \hat{a}_{m} \cdots a_{l}}^{A B, p-1} \\
& -\gamma^{*} \sum_{m=p+1}^{l}(-)^{m} \delta_{c a_{m}} \Omega_{a_{1} \cdots \hat{a}_{m} \cdots a_{l}}^{A B . p}
\end{aligned}
$$

where $\gamma=e^{i \pi / 4}$.

\section{B. Supersymmetric bilinears}

Among the bilinears constructed in the last subsection we have noted that $\rho^{A B}$ and the linear combination $\gamma \eta_{A B}^{b}-$ $\gamma^{*} \eta_{B A}^{b \dagger}$ are supersymmetric, i.e., they commute and anticommute, respectively, with $Q^{c}$. In fact they are just the first two of a sequence of such bilinears we can form from linear combinations of the $\Omega_{a_{1} \cdots a_{l}}^{A B, p}$. In the following we suppress the flavor labels $A, B$ since they are simply spectators.

For a given $l$, let $E^{a_{1} \cdots a_{l}}$ be a completely antisymmetric spinor-tensor of rank $l$. For $l=s$ the unique choice is the Levi-Cevita tensor. But for $l<s$ there are multiple choices. Then we calculate

$$
\begin{aligned}
{\left[Q^{c}, E^{a_{1} \cdots a_{l}} \Omega_{a_{1} \cdots a_{l}}^{p}\right]_{ \pm} } & =\gamma \sum_{m=1}^{p}(-)^{m+p} E^{a_{1} \cdots c \cdots a_{l}} \Omega_{a_{1} \cdots \hat{a}_{m} \cdots a_{l}}^{p-1}-\gamma^{*} \sum_{m=p+1}^{l}(-)^{m} E^{a_{1} \cdots c \cdots a_{l}} \Omega_{a_{1} \cdots \hat{a}_{m} \cdots a_{l}}^{p} \\
& =\gamma(-)^{p+1} p E^{c a_{1} \cdots a_{l-1}} \Omega_{a_{1} \cdots a_{l-1}}^{p-1}+\gamma^{*}(l-p) E^{c a_{1} \cdots a_{l-1}} \boldsymbol{\Omega}_{a_{1} \cdots a_{l-1}}^{p} .
\end{aligned}
$$

In the first line the index $c$ occupies the $m$ th location in the superscript of $E$. Then the last line is obtained by moving $c$ to the first location and relabeling the summation indices $a_{1} \cdots \hat{a}_{m} \cdots a_{l} \rightarrow a_{1} \cdots a_{l-1}$.

Now make the ansatz

$$
\Omega_{E_{l}}^{A B}=\sum_{p=0}^{l} c_{p} E^{a_{1} \cdots a_{l}} \Omega_{a_{1} \cdots a_{l}}^{A B, p}
$$

and require, still suppressing flavor indices, 


$$
\begin{aligned}
0=\left[Q^{c}, \Omega_{E}\right]_{ \pm}= & \sum_{p=0}^{l} c_{p}\left[\gamma(-)^{p+1} p E^{c a_{1} \cdots a_{l-1}} \Omega_{a_{1} \cdots a_{l-1}}^{p-1}\right. \\
& \left.+\gamma^{*}(l-p) E^{c a_{1} \cdots a_{l-1}} \Omega_{a_{1} \cdots a_{l-1}}^{p}\right] \\
= & \sum_{p=0}^{l-1}\left[c_{p+1} \gamma(-)^{p}(p+1)\right. \\
& \left.+c_{p} \gamma^{*}(l-p)\right] E^{c a_{1} \cdots a_{l-1}} \Omega_{a_{1} \cdots a_{l-1}}^{p}
\end{aligned}
$$

which implies the recursion relation

$$
c_{p+1}=-c_{p} \frac{(-)^{p} \gamma^{*}(l-p)}{\gamma(p+1)}
$$

with the solution, setting $c_{0}=\gamma^{l}$,

$$
c_{n}=(-)^{n(n+1) / 2} \gamma^{* n} \gamma^{l-n}\left(\begin{array}{l}
l \\
n
\end{array}\right) .
$$

\section{Supersymmetric quartics}

We now turn to the search for candidates for a supersymmetric string bit Hamiltonian, under the working hypothesis that $H$ will be quartic in the string bit creation and annihilation operators. The simplest possibilities are to take the products of pairs of the supersymmetric bilinears given in the previous section:

$$
: \Omega_{E_{l}}^{A B} \otimes \Omega_{F_{l^{\prime}}}^{C D}:
$$

is supersymmetric by construction. Except for $l, l^{\prime}=0$ or $=s, E$ and $F$ break the $O(s)$ symmetry, which could be restored either by suitable averages over $E, F$, or by adding new d.o.f. such as worldsheet coordinate fields, to compensate. Here, as before, the $\otimes$ symbol signifies that none of the suppressed color indices are contracted. Of course, since we require that the Hamiltonian be a color singlet, all these indices must be contracted. For example, the term in $H_{1}$ proportional to $s$ can be generalized to

$$
\frac{2}{N} \sum_{n=0}^{s} \sum_{k=0}^{s} \frac{s}{n ! k !} \operatorname{Tr} \bar{\phi}_{a_{1} \cdots a_{n}}^{A} \bar{\phi}_{b_{1} \cdots b_{k}}^{B} \phi_{b_{1} \cdots b_{k}}^{C} \phi_{a_{1} \cdots a_{n}}^{D}
$$

which can be obtained from $: \rho_{A D} \otimes \rho_{B C}$ : by the index contraction $:\left(\rho_{A D}\right)_{\alpha \delta}^{\beta \alpha}\left(\rho_{B G}\right)_{\beta \gamma}^{\gamma \delta}:$ It commutes with $Q^{a}$ since the latter commutes with $\rho_{A B}$. But any other contraction scheme will also preserve supersymmetry, and any such term can be included in modifying the Hamiltonian keeping supersymmetry. The normal ordering does not interfere with the product rule for commutators, because $Q^{a}$ is a one body operator.
Next we implement a similar generalization of $\mathrm{H}_{2-5}$.

$$
\begin{aligned}
& H_{2} \rightarrow \frac{2}{N} \sum_{n=0}^{s-1} \sum_{k=0}^{s-1} \frac{(-)^{k}}{n ! k !} \operatorname{Tr} \bar{\phi}_{a_{1} \cdots a_{n}}^{A} \bar{\phi}_{b b_{1} \cdots b_{k}}^{B} \phi_{b_{1} \cdots b_{k}}^{C} \phi_{b a_{1} \cdots a_{n}}^{D}, \\
& H_{3} \rightarrow \frac{2}{N} \sum_{n=0}^{s-1} \sum_{k=0}^{s-1} \frac{(-)^{k}}{n ! k !} \operatorname{Tr} \bar{\phi}_{b a_{1} \cdots a_{n}}^{A} \bar{\phi}_{b_{1} \cdots b_{k}}^{B} \phi_{b b_{1} \cdots b_{k}}^{C} \phi_{a_{1} \cdots a_{n}}^{D},
\end{aligned}
$$

$H_{4} \rightarrow \frac{2 i}{N} \sum_{n=0}^{s-1} \sum_{k=0}^{s-1} \frac{(-)^{k}}{n ! k !} \operatorname{Tr} \bar{\phi}_{a_{1} \cdots a_{n}}^{A} \bar{\phi}_{b_{1} \cdots b_{k}}^{B} \phi_{b b_{1} \cdots b_{k}}^{C} \phi_{b a_{1} \cdots a_{n}}^{D}$,

$$
H_{5} \rightarrow-\frac{2 i}{N} \sum_{n=0}^{s-1} \sum_{k=0}^{s-1} \frac{(-)^{k}}{n ! k !} \operatorname{Tr} \bar{\phi}_{b a_{1} \cdots a_{n}}^{A} \bar{\phi}_{b b_{1} \cdots b_{k}}^{B} \phi_{b_{1} \cdots b_{k}}^{C} \phi_{a_{1} \cdots a_{n}}^{D} .
$$

The right sides can be obtained, respectively, from

$$
\begin{array}{ll}
-\frac{2}{N}: \eta_{A D}^{b} \otimes \bar{\eta}_{B C}^{b}:, & \frac{2}{N}: \bar{\eta}_{A D}^{b} \otimes \eta_{B C}^{b}:, \\
-\frac{2 i}{N}: \eta_{A D}^{b} \otimes \eta_{B C}^{b}:, & -\frac{2 i}{N}: \bar{\eta}_{A D}^{b} \otimes \bar{\eta}_{B C}^{b}:
\end{array}
$$

Since the factor operators are not supersymmetric, the $H_{i}$ are not separately supersymmetric, but we shall see shortly that the sum of the modified terms is supersymmetric.

The commutator of $Q^{a}$ with each of these expressions is given, respectively, by

$$
\begin{aligned}
& \frac{2}{N}\left(\gamma: \eta_{A D}^{a} \otimes \rho_{B C}:-\gamma^{*}: \rho_{A D} \otimes \bar{\eta}_{B C}^{a}:\right), \\
& \frac{2}{N}\left(\gamma: \rho_{A D} \otimes \eta_{B C}^{a}:-\gamma^{*}: \bar{\eta}_{A D}^{a} \otimes \rho_{B C}:\right), \\
& \frac{2}{N}\left(\gamma: \eta_{A D}^{a} \otimes \rho_{B C}:-\gamma: \rho_{A D} \otimes \eta_{B C}^{a}:\right), \\
& \frac{2}{N}\left(\gamma^{*}: \rho_{A D} \otimes \bar{\eta}_{B C}^{a}:-\gamma^{*}: \bar{\eta}_{A D}^{a} \otimes \rho_{B C}:\right) .
\end{aligned}
$$

Notice that the sum of these four expressions is

$$
\frac{4}{N}\left(\gamma: \eta_{A D}^{a} \otimes \rho_{B C}:-\gamma^{*}: \bar{\eta}_{A D}^{a} \otimes \rho_{B C}:\right)
$$

which can be canceled by

$$
\frac{4}{N}\left[Q^{a},: \rho_{A D}^{\prime} \otimes \rho_{B C}:\right]=\frac{4}{N}:\left(\gamma \eta_{A D}^{a}-\gamma^{*} \bar{\eta}_{A B}^{a}\right) \otimes \rho_{B C}:
$$


Similarly, the sum of the first two lines minus the sum of the last two lines is

$$
\frac{4}{N}\left(\gamma: \rho_{A D}^{a} \otimes \eta_{B C}^{a}:-\gamma^{*}: \rho_{A D} \otimes \bar{\eta}_{B C}^{a}:\right)
$$

which can be canceled by

$$
\frac{4}{N}\left[Q^{a},: \rho_{A D} \otimes \rho_{B C}^{\prime}:\right]=\frac{4}{N}: \rho_{A D} \otimes\left(\gamma \eta_{B C}^{a}-\gamma^{*} \bar{\eta}_{B C}^{a}\right): .
$$

To summarize, in addition to the numerous supersymmetric quartics that can be written as products of supersymmetric bilinears, we have constructed two supersymmetric structures, as bilinears in the nonsupersymmetric bilinears $\eta^{a}$, $\bar{\eta}^{a}$, which enter the $H_{S}$ part of the original string bit Hamiltonian. We have not tried to find other such structures which are products of bilinears of tensors of higher rank, though we expect many more should exist. The structures entering $H_{S}$ are

$$
\begin{gathered}
\frac{2 s}{N}: \rho^{A D} \otimes \rho^{B C}: \\
\frac{2}{N}\left(-: \eta_{A D}^{b} \otimes \bar{\eta}_{B C}^{b}:+: \bar{\eta}_{A D}^{b} \otimes \eta_{B C}^{b}:-i: \eta_{A D}^{b} \otimes \eta_{B C}^{b}\right. \\
\left.-i: \bar{\eta}_{A D}^{b} \otimes \bar{\eta}_{B C}^{b}:-: \rho_{A D}^{\prime} \otimes \rho_{B C}\right), \\
\frac{2}{N}\left(-: \eta_{A D}^{b} \otimes \bar{\eta}_{B C}^{b}:+: \bar{\eta}_{A D}^{b} \otimes \eta_{B C}^{b}:+i: \eta_{A D}^{b} \otimes \eta_{B C}^{b}\right. \\
\left.+i: \bar{\eta}_{A D}^{b} \otimes \bar{\eta}_{B C}^{b}:-: \rho_{A D} \otimes \rho_{B C}^{\prime}\right),
\end{gathered}
$$

where the first is a product of supersymmetric bilinears, and the last two are (at least apparently) not of this type. To build a supersymmetric Hamiltonian from these structures, simply multiply each of these forms by an independent $T_{k}^{A B C D}$ and sum over all flavors. All color indices need to be contracted, but supersymmetry holds for all contraction schemes.

\section{APPLICATIONS TO THE IIB SUPERSTRING}

In [13] the connection of string bit models to string formation has been established in the limit $N \rightarrow \infty$, which corresponds to zero string coupling. In that limit $H_{S}$ on multitrace states acts independently on each trace with only nearest neighbor interactions of the bits on the trace, identical with those of a discretized lightcone quantized Green-Schwarz Grassmann world sheet field. Interactions between these discretized strings are present in $H_{S}$ at order $1 / N$, but they do not provide the operator prefactor required for Poincare invariance in the continuum limit. To describe the IIB superstring, additional terms must be added to $H_{S}$ which supply these prefactors at order $1 / N$, while not contributing at $N=\infty$.
In this section we determine supersymmetric terms, involving the $\Omega^{p}$ discussed in the previous section, which can provide the necessary prefactors. In addition to the eight pairs of Grassmann worldsheet fields $S^{a}(\sigma, \tau)$, $\tilde{S}^{a}(\sigma, \tau)$, the superstring requires eight bosonic transverse coordinates $x^{k}(\sigma, \tau)$. In [13] we argued that these transverse coordinates might actually be merely effective fields, which describe the low energy dynamics of long Heisenberg spin chains, in which the spin matrices act on internal d.o.f., which we have called "flavor." However for the purposes of this discussion we take them as fundamental worldsheet fields rather than effective ones. Green, Schwarz, and Brink [19] derived the vertex insertion required by Poincaré invariance. It has the structure

$$
\tilde{X}^{j}\left(\sigma_{I}, \tau_{I}\right) X^{k}\left(\sigma_{I}, \tau_{I}\right) V_{j k}\left(Y\left(\sigma_{I}, \tau_{I}\right)\right)
$$

with $V_{j k}$ a linear combination of the five structures

$$
\begin{aligned}
& \delta_{j k}, \quad \gamma_{a b}^{j k} Y^{a} Y^{b}, \quad t_{a b c d}^{j k} Y^{a} Y^{b} Y^{c} Y^{d}, \\
& \gamma_{a b}^{j k} \epsilon^{a b c d e f g h} Y^{c} Y^{d} Y^{e} Y^{f} Y^{g} Y^{h}, \\
& \delta_{j k} \epsilon^{a b c d e f g h} Y^{a} Y^{b} Y^{c} Y^{d} Y^{e} Y^{f} Y^{g} Y^{h} .
\end{aligned}
$$

In these expressions $\tilde{X}, X$ are left and right moving derivatives of the transverse coordinate worldsheet fields $x^{k}(\sigma, \tau)$ :

$$
X(\tilde{X}) \propto \lim _{\sigma \rightarrow \sigma_{I}} \sqrt{\sigma_{I}-\sigma}\left[\mathcal{P}(\sigma) \mp x^{\prime}(\sigma)\right]
$$

where $\mathcal{P}=\dot{x}$ is the momentum density conjugate to $x$ and where the limit is taken at the end of the calculation. Also the point on the worldsheet where a single string separates into two strings is marked by $\sigma_{I}, \tau_{I}$. The square root factor is necessary because the interaction point is singular, making the insertion blow up exactly at $\sigma=\sigma_{I}$. This is a drawback of trying to work with a continuous world sheet. This drawback is not present in the string bit approach because in those models the emergent worldsheet is discretized and behaves as a continuous worldsheet only for low energy excitations. In string bit models the $X$ or $\tilde{X}$ insertion is simply $p_{k} \pm\left(x_{k+1}-x_{k}\right)$. The $Y^{a}$ are linear combinations of the Grassmann worldsheet fields.

$$
Y \propto \lim _{\sigma \rightarrow \sigma_{I}} \sqrt{\sigma_{I}-\sigma}[\theta(\sigma)+\delta / \delta \theta(\sigma)] .
$$

Again, the limiting procedure is necessary because the worldsheet is continuous. In this case it gives the misleading impression that the different powers of $Y$ in the complete insertion have different divergent behavior. But, as shown in [14], because the relation of a discretized local Grassmann variable such as $\theta_{k}$ to energy ladder operators $B_{n}$ is of the form 


$$
\theta_{k} \sim \frac{1}{\sqrt{M}} \sum_{n=0}^{M-1} e^{2 \pi i n k / M} B_{n}
$$

the square root divergence $(\sim \sqrt{M})$ indicated by (64) is precisely what is needed to leave a finite nonzero effect in the continuum limit $(M \rightarrow \infty)$, when the insertion is applied to energy eigenstates. In string bit models the $Y$ dependent factor of the insertion will simply be sums of powers of a linear combination of $\theta_{k}$ and $\delta / \delta \theta_{k}$, with no further regulation.

We now show how these structures can be generated by supersymmetric terms added to the string bit Hamiltonian. We start by working out the commutator of $\Omega_{E_{l}}^{A B, p}$ with the superstring bit creation operator $\bar{\psi}^{C}(\theta)$.

$$
\begin{aligned}
& {\left[\Omega_{E_{l}}^{A B, p}, \bar{\psi}^{C}(\theta)\right] } \\
= & \delta_{B C} E^{a_{1} \cdots a_{l}} \sum_{k} \frac{(-)^{k l}}{k !} \bar{\phi}_{a_{1} \cdots a_{p} b_{1} \cdots b_{k}}^{A} \theta^{a_{p+1}} \cdots \theta^{a_{l}} \theta^{b_{1}} \cdots \theta^{b_{k}} \\
= & \delta_{B C} E^{a_{1} \cdots a_{l}}(-)^{p(l-p)} \theta^{a_{p+1}} \cdots \theta^{a_{l}} \\
& \times \sum_{k} \frac{(-)^{k p}}{k !} \bar{\phi}_{a_{1} \cdots a_{p} b_{1} \cdots b_{k}}^{A} \theta^{b_{1}} \cdots \theta^{b_{k}}
\end{aligned}
$$

To express the right side in terms of $\bar{\psi}(\theta)$ we calculate

$\frac{d}{d \theta^{a_{p}}} \cdots \frac{d}{d \theta^{a_{1}}} \bar{\psi}^{A}(\theta)=\sum_{k=0}^{s} \frac{(-)^{p(p+k)}}{k !} \bar{\phi}_{a_{1} \cdots a_{p} b_{1} \cdots b_{k}}^{A} \theta^{b_{1}} \cdots \theta^{b_{k}}$.
The sum on the right is proportional to the sum appearing in the commutator, so we can write

$$
\begin{aligned}
{\left[\Omega_{E_{l}}^{A B, p}, \bar{\psi}^{C}(\theta)\right]=} & \delta_{B C} E^{a_{1} \cdots a_{l}}(-)^{p l} \theta^{a_{p+1}} \cdots \theta^{a_{l}} \frac{d}{d \theta^{a_{p}}} \cdots \frac{d}{d \theta^{a_{1}}} \\
& \times \psi^{A}(\theta) .
\end{aligned}
$$

Finally we reorder the indices of $E$ to match the ordering of the $\theta$ and $d / d \theta$ factors

$$
E^{a_{1} \cdots a_{l}}=(-)^{p(l-p)+p(p-1) / 2} E^{a_{p+1} \cdots a_{l} a_{p} \cdots a_{1}}
$$

and then rename them to get

$$
\begin{aligned}
{\left[\Omega_{E_{l}}^{A B, p}, \bar{\psi}^{C}(\theta)\right]=} & \delta_{B C} E^{a_{1} \cdots a_{l}}(-)^{p(p+1) / 2} \theta^{a_{1}} \cdots \theta^{a_{l-p}} \\
& \times \frac{d}{d \theta^{a_{l-p+1}}} \cdots \frac{d}{d \theta^{a_{l}}} \bar{\psi}^{A}(\theta) / .
\end{aligned}
$$

A clarification is needed about suppressed color indices in this equation. The color indices can all be left uncontracted. In that case there is a suppressed Kronecker delta $\delta_{\alpha}^{\delta} \delta_{\gamma}^{\beta}$ on the right side which requires the color indices of $\left(\bar{\psi}^{C}\right)_{\alpha}^{\beta}$ to match the color indices of the factor $\left(\phi^{B}\right)_{\gamma}^{\delta}$ in $\Omega^{A B}$. Then the color indices of the factor $\bar{\phi}^{A}$ are identical to those of the $\bar{\psi}^{A}$ on the right side. With this in mind one can choose to do any number of contractions on the color indices without spoiling the validity of the equation.

Now one finds, for the supersymmetric combination

$$
\begin{aligned}
{\left[\Omega_{E_{l}}^{A B}, \bar{\psi}^{C}(\theta)\right] } & =\gamma^{l} \delta_{B C} E^{a_{1} \cdots a_{l}} \sum_{p=0}^{l}\left(\frac{\gamma^{*}}{\gamma}\right)^{p}\left(\begin{array}{l}
l \\
p
\end{array}\right) \theta^{a_{1}} \cdots \theta^{a_{l-p}} \frac{d}{d \theta^{a_{l-p+1}}} \cdots \frac{d}{d \theta^{a_{l}}} \bar{\psi}^{A}(\theta) \\
& =\gamma^{l} \delta_{B C} E^{a_{1} \cdots a_{l}}\left(\theta^{a_{1}}+\frac{\gamma^{*}}{\gamma} \frac{d}{d \theta^{a_{1}}}\right) \cdots\left(\theta^{a_{l}}+\frac{\gamma^{*}}{\gamma} \frac{d}{d \theta^{a_{l}}}\right) \bar{\psi}^{A}(\theta) \\
& =\delta_{B C} E^{a_{1} \cdots a_{l}}\left(\gamma \theta^{a_{1}}+\gamma^{*} \frac{d}{d \theta^{a_{1}}}\right) \cdots\left(\gamma \theta^{a_{l}}+\gamma^{*} \frac{d}{d \theta^{a_{l}}}\right) \bar{\psi}^{A}(\theta) .
\end{aligned}
$$

A quick check on this final answer is to note that the action of $Q^{a}$ on $\bar{\psi}^{C}$ is given by

$$
\left[Q^{a}, \bar{\psi}^{C}\right]_{ \pm}=\left(\gamma \theta^{a}-\gamma^{*} \frac{d}{d \theta^{a}}\right) \bar{\psi}^{C}
$$

and the differential operator on the right anticommutes with each of the factor operators in the action of $\Omega_{E_{l}}^{A B}$ :

$$
\left\{\gamma \theta^{a}-\gamma^{*} \frac{d}{d \theta^{a}}, \gamma \theta^{b}+\gamma^{*} \frac{d}{d \theta^{b}}\right\}=0,
$$

which is necessarily true if $\Omega_{E_{l}}^{A B}$ is indeed a supersymmetric operator.
We have shown that $\Omega_{E_{l}}^{A B}$ can produce the insertion operators required for the IIB superstring. It remains to use them to construct color singlet terms to be added to the Hamiltonian such that they do not contribute at leading order as $N \rightarrow \infty$, but they do contribute at order $1 / N$ in such a way that the necessary operators accompany each closed string fission or fusion transition. The way to do this is to construct color singlet quartic operators with the schematic color structure

$$
\frac{1}{N} \operatorname{Tr}: \bar{\phi} \phi \bar{\phi} \phi:=\frac{1}{N}:(\bar{\phi})_{\alpha}^{\beta}(\phi)_{\beta}^{\gamma}(\bar{\phi})_{\gamma}^{\delta}(\phi)_{\delta}^{\alpha}:
$$

where the color indices are contracted as in matrix multiplication. The normal ordering removes the $O(1)$ 
contraction, which would arise if the normal ordering were not specified. The terms dropped by normal ordering would be supersymmetric bilinears which could be included independently of the quartic terms, if desired.

Acting on multitrace states this operator does not contribute at leading order because contraction of the two annihilation operators will not produce a factor of $N$ to compensate the $1 / N$ out front. Furthermore any such contraction will change the trace structure of the state, splitting a trace into two traces, or joining two traces into one.

To build the desired terms we first contract two of the color indices of $\Omega_{E_{l}}^{A B}$ to form a matrix $\vec{\Omega}_{E_{l}}^{A B}$. Then we multiply this operator with $\vec{\rho}^{C D}$ and take the trace to form the color singlet

$$
\frac{1}{N} \operatorname{Tr}: \vec{\Omega}_{E_{l}}^{A B} \vec{\rho}^{C D}:
$$

By construction this is a supersymmetric color singlet operator with the appropriate color contraction scheme to first contribute at order $1 / N$ in the large $N$ expansion. We confirm this by applying it to a multitrace state. On a single trace state we have, consulting (71)

$$
\begin{aligned}
\frac{1}{N} \operatorname{Tr}: \vec{\Omega}_{E_{l}}^{A B} \vec{\rho}^{C D}: T\left(A_{1} \theta_{1} ; \cdots ; A_{M} \theta_{M}\right)|0\rangle= & \frac{1}{N} \sum_{k, m}\left[\delta_{D A_{k}} \delta_{B A_{m}} ; T\left(C \theta_{k} ; \cdots ; A_{m-1} \theta_{m-1}\right)\right. \\
& \left.\times E^{a_{1} \cdots a_{l}} \Lambda_{m}^{a_{1}} \cdots \Lambda_{m}^{a_{l}} T\left(A \theta_{m} ; A_{m+1} \cdots ; A_{k-1} \theta_{k-1}\right)\right]|0\rangle
\end{aligned}
$$

where

$$
\Lambda_{m}^{a}=\gamma \theta_{m}^{a}+\gamma^{*} \frac{d}{d \theta_{m}^{a}}
$$

We see that the action has split the trace into two traces and the $1 / N$ factor is uncanceled. For $l=0,2,4,6,8$ the action of this operator produces the five insertions listed in (62) respectively.

The fusion of two traces into one is also produced by this operator acting on a state containing two or more traces. This happens when the two annihilation operators contract against distinct traces,

$$
\begin{aligned}
& \frac{1}{N} \operatorname{Tr}: \vec{\Omega}_{E_{l}}^{A B} \vec{\rho}^{C D}: T\left(A_{1} \theta_{1} \cdots A_{K} \theta_{K}\right) T\left(B_{1} \eta_{1} \cdots B_{L} \eta_{L}\right)|0\rangle_{\text {Fusion }} \\
& =\frac{1}{N} \sum_{k=1}^{K} \sum_{m=1}^{L} \delta_{D A_{k}} \delta_{B B_{m}} E^{a_{1} \cdots a_{l}} \Lambda_{\eta_{m}}^{a_{1}} \cdots \Lambda_{\eta_{m}}^{a_{l}} T\left(C \theta_{k} \cdots A_{k-1} \theta_{k-1} A \eta_{m} \cdots B_{m-1} \eta_{m-1}\right)|0\rangle \\
& \quad+\frac{1}{N} \sum_{k=1}^{K} \sum_{l=1}^{L} \delta_{D B_{l}} \delta_{B A_{k}} E^{a_{1} \cdots a_{l}} \Lambda_{\theta_{k}}^{a_{1}} \cdots \Lambda_{\theta_{k}}^{a_{l}} T\left(A \theta_{k} \cdots A_{k-1} \theta_{k-1} C \eta_{m} \cdots B_{m-1} \eta_{m-1}\right)|0\rangle
\end{aligned}
$$

If supersymmetry were the only requirement for modifying the Hamiltonian, we could form $J_{A B C D} \operatorname{Tr}: \vec{\Omega}_{E_{l}}^{A B} \vec{\rho}^{C D}$ : with $J_{A B C D}$ arbitrary complex numbers and add it plus its Hermitian conjugate to the Hamiltonian. A simple choice for $J$ which works for the IIB superstring is $J_{A B C D}=\delta_{A B} \delta_{C D}$.

For the IIB superstring, the antisymmetric tensors $E_{l}$ are built from $S O(8)$ gamma matrices [19]. For even $l$, as required in (62), they are second rank tensors in $S O(8)$ vector indices, $E_{2 l}^{k l}$, which can read off from (62). The relative coefficients in the complete insertion operator cannot be taken directly from [19], because in the latter reference the continuum limit has already been partially taken, holding the insertion a fixed distance from the interaction point. The string bit approach is discrete at a fundamental level, and the continuum limit is properly regarded as a low excitation energy approximation on chains with large numbers of bits, which must be carefully analyzed to make a detailed comparison. The terms we have constructed will prescribe that the insertion is within one or two discrete units away from the location of the separation or joining point, whereas the approach of [19] would correspond to placing the insertion $\epsilon M$, with $\epsilon$ small but fixed, units away. Such a prescription would be far from natural in the string bit approach. The continuum limits in these two methods are not taken in the same way. Although it seems plausible that term by term the two methods will give results that are proportional, it would be premature to expect that the proportionality constants be exactly one.

\section{CONCLUSION}

We have found many ways to modify the string bit Hamiltonian of [13] while maintaining supersymmetry. 
In particular, we have constructed explicit supersymmetric operators which, added to the Hamiltonian, can provide the insertion prefactors necessary for the overlap prescription for lightcone superstring vertices to give correct scattering amplitudes. This ties up one of the loose ends in [13]. In this discussion we have treated the coordinate worldsheet fields as fundamental rather than as effective fields simulating low energy excitations of a discrete flavor variable. In [13] we showed that a system of Heisenberg chains described by such an effective field can be obtained in the $N \rightarrow \infty$ limit from the dynamics of $2^{d}$ valued "flavor" indices. It will be interesting to pursue this idea further by extending it to interactions. Specifically, understanding how the $\tilde{X}^{k} X^{l}$ factor in the prefactor (61) can be obtained from such a discrete flavor dynamics is a natural next step.

\section{ACKNOWLEDGMENTS}

I would like to thank Ethan Torres for his early contributions to this project and for valuable discussions. This research was supported in part by the Department of Energy under Grant No. DE-SC0010296.

APPENDIX: PROOF OF EQ. (38)

The first three lines of Eq. (A1) arise from the bracket of $Q$ with $\bar{\phi}$ and the last three lines from that of $Q$ with $\phi$,

$$
\begin{aligned}
{\left[Q^{c}, \Omega_{a_{1} \cdots a_{l}}^{p}\right]=} & \sum_{k} \frac{(-)^{l k}}{k !}\left[(-)^{k+p} \gamma^{*} \bar{\phi}_{c a_{1} \cdots a_{p} b_{1} \cdots b_{k}} \phi_{a_{p+1} \cdots a_{l} b_{1} \cdots b_{k}}\right. \\
& +\gamma \sum_{m=1}^{p}(-)^{m+k+p} \delta_{c a_{m}} \bar{\phi}_{a_{1} \cdots \hat{a}_{m} \cdots a_{p} b_{1} \cdots b_{k}} \phi_{a_{p+1} \cdots a_{l} b_{1} \cdots b_{k}} \\
& \left.+\gamma \sum_{m=1}^{k}(-)^{m+k+1} \delta_{c b_{m}} \bar{\phi}_{a_{1} \cdots a_{p} b_{1} \cdots \hat{b}_{m} \cdots b_{k}} \phi_{a_{p+1} \cdots a_{l} b_{1} \cdots b_{k}}\right] \\
& +\sum_{k} \frac{(-)^{l k}}{k !}\left[(-)^{k+p+1} \bar{\phi}_{a_{1} \cdots a_{p} b_{1} \cdots b_{k}} \phi_{c a_{p+1} \cdots a_{l} b_{1} \cdots b_{k}}\right. \\
& +\gamma^{*} \sum_{m=1}^{l-p}(-)^{m+k+p+1} \delta_{c a_{m+p}} \bar{\phi}_{a_{1} \cdots a_{p} b_{1} \cdots b_{k}} \phi_{a_{p+1} \cdots \hat{a}_{m+p} \cdots a_{l} b_{1} \cdots b_{k}} \\
& \left.+\gamma^{*} \sum_{m=1}^{k}(-)^{m+k+l+1} \delta_{c b_{m}} \bar{\phi}_{a_{1} \cdots a_{p} b_{1} \cdots b_{k}} \phi_{a_{p+1} \cdots a_{l} b_{1} \cdots \hat{b}_{m} \cdots b_{k}}\right]
\end{aligned}
$$

The first line is canceled by the sixth line. To see this use the Kronecker delta of the latter to set $b_{m}=c$, and then use the antisymmetry in indices. Then the summand is independent of $m$, so the sum over $m$ just gives a factor of $k$, which changes $1 / k ! \rightarrow 1 /(k-1)$ !. Then shifting $k \rightarrow k+1$ gives - line 1 . Similarly line 3 cancels line 4 . Lines 2 and 5 are all that are left:

$$
\begin{aligned}
{\left[Q^{c}, \Omega_{a_{1} \cdots a_{l}}^{p}\right]=} & \sum_{k} \frac{(-)^{l k}}{k !}\left[+\gamma \sum_{m=1}^{p}(-)^{m+k+p} \delta_{c a_{m}} \bar{\phi}_{a_{1} \cdots \hat{a}_{m} \cdots a_{p} b_{1} \cdots b_{k}} \phi_{a_{p+1} \cdots a_{l} b_{1} \cdots b_{k}}\right. \\
& \left.+\gamma^{*} \sum_{m=1}^{l-p}(-)^{m+k+p+1} \delta_{c a_{m+p}} \bar{\phi}_{a_{1} \cdots a_{p} b_{1} \cdots b_{k}} \phi_{a_{p+1} \cdots \hat{a}_{m+p} \cdots a_{l} b_{1} \cdots b_{k}}\right] .
\end{aligned}
$$

The two sums over $k$ can now be recognized as two of the $\Omega$ 's:

$$
\left[Q^{c}, \Omega_{a_{1} \cdots a_{l}}^{p}\right]=+\gamma \sum_{m=1}^{p}(-)^{m+p} \delta_{c a_{m}} \Omega_{a_{1} \cdots \hat{a}_{m} \cdots a_{l}}^{p-1}+\gamma^{*} \sum_{m=p+1}^{l}(-)^{m+1} \delta_{c a_{m}} \Omega_{a_{1} \cdots \hat{a}_{m} \cdots a_{l}}^{p}
$$

which is (38). 
[1] C. B. Thorn, Reformulating string theory with the $1 / \mathrm{N}$ expansion, arXiv:hep-th/9405069.

[2] G. 't Hooft, Quantization of discrete deterministic theories by Hilbert space extension, Nucl. Phys. B342, 471 (1990); On the quantization of space and time, in Proceedings of the 4th Seminar on Quantum Gravity, 1987, Moscow, USSR, edited by M. A. Markov (World Scientific Press, Singapore, 1988); Dimensional reduction in quantum gravity, Conf. Proc. C930308, 284 (1993), https://arxiv.org/abs/gr-qc/9310026.

[3] P. Goddard, J. Goldstone, C. Rebbi, and C. B. Thorn, Quantum dynamics of a massless relativistic string, Nucl. Phys. B56, 109 (1973).

[4] S. Mandelstam, Interacting string picture of dual resonance models, Nucl. Phys. B64, 205 (1973); Interacting string picture of the Neveu-Schwarz-Ramond model, Nucl. Phys. B69, 77 (1974).

[5] R. Giles and C. B. Thorn, A lattice approach to string theory, Phys. Rev. D 16, 366 (1977).

[6] G. 't Hooft, A planar diagram theory for strong interactions, Nucl. Phys. B72, 461 (1974).

[7] C. B. Thorn, A fock space description of the $1 / N_{c}$ expansion of quantum chromodynamics, Phys. Rev. D 20, 1435 (1979).

[8] P. Ramond, Dual theory for free Fermions, Phys. Rev. D 3, 2415 (1971); A. Neveu and J. H. Schwarz, Factorizable dual model of pions, Nucl. Phys. B31, 86 (1971); A. Neveu, J. H. Schwarz, and C. B. Thorn, Reformulation of the dual Pion model, Phys. Lett. B 35, 529 (1971); C. B. Thorn,
Embryonic dual model for Pions and Fermions, Phys. Rev. D 4, 1112 (1971); A. Neveu and J. H. Schwarz, Quark model of dual Pions, Phys. Rev. D 4, 1109 (1971).

[9] F. Gliozzi, J. Scherk, and D. I. Olive, Supersymmetry, supergravity theories and the dual Spinor model, Nucl. Phys. B122, 253 (1977).

[10] M. B. Green and J.H. Schwarz, Supersymmetrical dual string theory, Nucl. Phys. B181, 502 (1981); K. Bardakci and M. B. Halpern, New dual quark models, Phys. Rev. D 3, 2493 (1971).

[11] O. Bergman and C. B. Thorn, String bit models for superstring, Phys. Rev. D 52, 5980 (1995).

[12] S. Sun and C. B. Thorn, Stable string bit models, Phys. Rev. D 89, 105002 (2014).

[13] C. B. Thorn, Space from string bits, J. High Energy Phys. 11 (2014) 110.

[14] C. B. Thorn, 1/N perturbations in superstring bit models, Phys. Rev. D 93, 066003 (2016).

[15] G. Chen and S. Sun, Numerical study of the simplest string bit model, Phys. Rev. D 93, 106004 (2016).

[16] G. Chen, Cubic interaction vertices and one-loop selfenergy in the stable string bit model, Phys. Rev. D 96, 046021 (2017).

[17] S. Sun, Aspects of stable string bit models, Ph. D. thesis, University of Florida, 2019.

[18] E. Torres (private communication).

[19] M. B. Green, J. H. Schwarz, and L. Brink, Superfield theory of type II superstrings, Nucl. Phys. B219, 437 (1983). 